\title{
Erratum
}

\section{Retrodictive states and two-photon quantum imaging}

\author{
E.-K. $\operatorname{Tan}^{1, a}$, J. Jeffers ${ }^{1}$, S.M. Barnett ${ }^{1}$, and D.T. Pegg ${ }^{2}$ \\ 1 Department of Physics, University of Strathclyde, John Anderson Building, 107 Rottenrow, Glasgow G4 0NG, UK \\ 2 School of Science, Griffith University, Nathan, Brisbane, Queensland Q 111, Australia
}

Received 8 February 2004

Published online 23 March 2004 - (c) EDP Sciences, Società Italiana di Fisica, Springer-Verlag 2004

Eur. Phys. J. D 22, 495-499 (2003)

Some of the detail in Section 3 of Eur. Phys. J. D 22, 495 (2003) are incorrect. However, the main idea of the paper still holds and our principal conclusions remain valid. The corrections are listed as below.

(I) To be consistent with the standard predictive treatment, the object transmission function $t$ in equations (7, 8 , 21 ) and the fourth line after equation (20) should be $t^{*}$. On the other hand, it should be $t$ in equation (24).

(II) Equation (19) should be replaced by the equation

$$
\tilde{\alpha}_{1}\left(k_{x}\right)=\mathrm{e}^{-\mathrm{i} k\left(z_{1}+f\right)}\left(\frac{\mathrm{i} f}{\pi^{1 / 2} k \sigma}\right)^{1 / 2} \exp \left[-\frac{x_{1}^{2}}{2 \sigma^{2}}+\left(\frac{\mathrm{i}\left(z_{1}-f\right)}{2 k}-\frac{f^{2}}{2 k^{2} \sigma^{2}}\right) k_{x}^{2}+\frac{f x_{1} k_{x}}{k \sigma^{2}}\right] .
$$

(III) Equation (20) should be replaced by the equation

$$
\alpha_{1}(x)=\mathrm{e}^{-\mathrm{i} k\left(z_{1}+f\right)}\left(\frac{\mathrm{i} 2 \pi^{1 / 2} \sigma}{\lambda \eta z_{1}\left(\frac{1}{\eta}+\frac{1}{z_{1}}-\frac{1}{f}\right)}\right)^{1 / 2} \exp \left[-\frac{\mathrm{i} \pi}{\lambda}\left(\frac{x_{1}^{2}}{\eta}+\frac{x^{2}}{z_{1}}\right)+\frac{\mathrm{i} \pi}{\lambda\left(\frac{1}{\eta}+\frac{1}{z_{1}}-\frac{1}{f}\right)}\left(\frac{x_{1}}{\eta}+\frac{x}{z_{1}}\right)^{2}\right]
$$

where $\eta=f+\mathrm{i} k \sigma^{2}$.

(IV) Equation (25) should be replaced by the equation

$$
\beta_{2}(x)=\mathrm{e}^{\mathrm{i} k\left(z_{2}+f\right)}\left(\frac{f}{2 \pi\left(f-z_{2}\right)}\right)^{1 / 2} \int \mathrm{d} k_{x} \tilde{\beta}_{1}\left(k_{x}\right) \exp \left[\frac{\mathrm{i} f^{2}}{2 k\left(z_{2}-f\right)}\left(k_{x}-\frac{k}{f} x\right)^{2}\right] .
$$

(V) $z_{1}$ and $z_{2}$ are taken into account because they affect the spatial profile in the transverse detection plane in arm 2 . For the case where $z_{1}=z_{2}=f$ and the limit of $\sigma, \kappa \rightarrow 0$, the transverse probability distribution for detection in arm 2 takes on the form of the squared modulus of the spatial Fourier transform of $t(x)$. On the other hand, for the same configuration in arm 1 , if the crystal and detector are both positioned at a distance $2 f$ from the lens in arm 2, the transverse probability distribution in arm 2 will be proportional to $|t(x)|^{2}$.

\footnotetext{
a e-mail: peter@phys.strath.ac.uk
} 